\title{
Variability in stellar granulation and convective blueshift with spectral type and magnetic activity
}

\section{From young to old main-sequence K-G-F stars ${ }^{\star}$}

\begin{abstract}
N. Meunier, L. Mignon, and A.-M. Lagrange
Univ. Grenoble Alpes, CNRS, IPAG, 38000 Grenoble, France

e-mail: nadege.meunier@univ-grenoble-alpes.fr

Received 21 April 2017 / Accepted 29 August 2017

\section{ABSTRACT}

Context. The inhibition of small-scale convection in the Sun dominates the long-term radial velocity (RV) variability: it therefore has a critical effect on light exoplanet detectability using RV techniques.

Aims. We here extend our previous analysis of stellar convective blueshift and its dependence on magnetic activity to a larger sample of stars in order to extend the $T_{\text {eff }}$ range, to study the impact of other stellar properties, and finally to improve the comparison between observed RV jitter and expected RV variations.

Methods. We estimate a differential velocity shift for $\mathrm{Fe}$ and Ti lines of different depths and derive an absolute convective blueshift using the Sun as a reference for a sample of 360 F7-K4 stars with different properties (age, $T_{\text {eff }}$, metallicity).

Results. We confirm the strong variation in convective blueshift with $T_{\text {eff }}$ and its dependence on (as shown in the line list in Paper I) activity level. Although we do not observe a significant effect of age or cyclic activity, stars with a higher metallicity tend to have a lower convective blueshift, with a larger effect than expected from numerical simulations. Finally, we estimate that for $71 \%$ of the stars in our sample the RV and $\log R_{\mathrm{HK}}^{\prime}$ variations are compatible with the effect of activity on convection, as observed in the solar case, while for the other stars, other sources (such as binarity or companions) must be invoked to explain the large RV variations. We also confirm a relationship between $\log R_{\mathrm{HK}}^{\prime}$ and metallicity, which may affect discussions of the possible relationship between metallicity and exoplanets, as RV surveys are biased toward low $\log R_{\mathrm{HK}}^{\prime}$ and possibly toward high-metallicity stars.

Conclusions. We conclude that activity and metallicity strongly affect the small-scale convection levels in stars in the F7-K4 range, with a lower amplitude for the lower mass stars and a larger amplitude for low-metallicity stars.
\end{abstract}

Key words. convection - techniques: radial velocities - stars: magnetic field - stars: activity - stars: solar-type - Sun: granulation

\section{Introduction}

Meunier et al. (2017, hereafter Paper I), measured the amplitude of the convective blueshift for a large sample of stars. This covered $\mathrm{G}$ and $\mathrm{K}$ main-sequence stars for six spectral types, $\mathrm{K} 2, \mathrm{~K} 0$, G8, G5, G2, and G0. The authors observed a strong decrease toward low-mass stars. Furthermore, we showed for the first time a significant dependence of the convective blueshift on the activity level. We found that the attenuation factor of the convective blueshift in solar-like plages (due to a higher concentration of the magnetic field) seems to follow a constant law as a function of spectral type. These results are crucial to characterize the effect of stellar activity on radial velocity (RV) measurements more precisely, and following from this, the effect on exoplanet detectability (Meunier et al. 2010). In particular, the results enable us to extrapolate the simulations made by Borgniet et al. (2015) to stars other than the Sun.

In this new study, we extend our sample by more than a factor of two (leading to 360 stars) with three main objectives: i) extension toward higher-mass stars (up to F7 stars) and lower-mass stars (down to K4 stars) to extend the validity domain of our conclusions for both the dependence on spectral type and activity level; ii) study the dependence of the convective blueshift

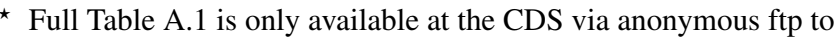
cdsarc.u-strasbg. fr (130.79.128.5) or via

http://cdsarc.u-strasbg.fr/viz-bin/qcat?J/A+A/607/A124
}

on other stellar properties, such as the type of variability (cyclic behavior or more stochastic variability), on age, and on metallicity, especially for solar-like stars, of which we have been able to select a large sample; iii) improvement of the comparison between the RV variability due to the attenuation of the convective blueshift with observations, based on a careful analysis of the relationship between RV and $\log R_{\mathrm{HK}}^{\prime}$ variations in our sample, from which we derive some statistics. The sample studied in Paper I was restricted to relatively old main-sequence stars. However, we know that the activity patterns of younger stars are different from the Sun, with a stronger influence caused by spots over plages (e.g., Lockwood et al. 2007). Plages are much more common in these stars than in the Sun, however, as shown by the much larger $\log R_{\mathrm{HK}}^{\prime}$ index, and it is not known whether their properties are different from solar properties. Moreover, numerical simulations have shown an effect of metallicity on convection that is due to the differences in opacities, although with some unclear pattern (Magic et al. 2013, 2014; Allende Prieto et al. 2013; Tremblay et al. 2013).

As in Paper I, our analysis is based on the estimation of the differential velocity shift (i.e., computed over a set of lines of different fluxes), as previously done for the Sun (Dravins et al. 1981; Hamilton \& Lester 1999) and other stars (Gray 1982; Dravins 1987, 1999; Landstreet 2007; Allende Prieto et al. 2002; Gray 2009; Meunier et al. 2017). This is then converted into an absolute convective blueshift using the Sun as a reference, as in 
Table 1. Sample origin.

\begin{tabular}{clll}
\hline \hline$\#$ & Reference & Type of survey & Number of stars \\
\hline 1 & Sousa et al. (2008) & F-G-K stars, old, not very active, exoplanet survey & 294 \\
2 & Ramírez et al. (2014) & Solar twins, old and young stars & 64 \\
3 & Marsden et al. (2014) & Solar-type stars, old and young (Bcool survey) & 31 \\
4 & Datson et al. (2014) & Solar twins & 5 \\
5 & Borgniet et al. (2017) & F stars, old stars, exoplanet survey & 6 \\
6 & Lagrange et al. (2013) & Young stars, exoplanet survey & 8 \\
7 & Gray et al. (2015) & Young solar twins & 4 \\
\hline
\end{tabular}

Notes. Number of stars retrieved from these surveys to build our sample.

Paper I. The absolute convective blueshift varies from one line to the other and also depends on how it was measured. In the following, the reference to an absolute convective blueshift corresponds to a single value (see in particular Sect. 2.2) associated with a certain set of lines, following the procedure described in Paper I. This therefore needs to be kept in mind when attempting to compare absolute values with other works. We focus our analysis on long-term variability rather than on variability at the rotational timescale, as has been done by Suárez Mascareño et al. (2017), and we also study a much larger sample. We note we focus here on the line shifts and not on the asymmetries of the lines that are due to convection or other effects such as rotation (Gray 1986).

The outline of the paper is the following. In Sect. 2 we describe our sample, and then summarize our method (we refer to Meunier et al. 2017, Paper I, for more details). In Sect. 3 we analyze the dependence of the convective blueshift on spectral type (using $B-V$ and $T_{\text {eff }}$ ), on the activity level, on the type of variability, on age, and on metallicity. In Sect. 4 we derive statistical information on how the RV variations correlate with activity variations. This information allows us to estimate the effect that the inhibited convection blueshift has on stars other than the Sun, for which we know it is a dominating factor. Finally, we conclude in Sect. 5.

\section{Data analysis}

\subsection{Star sample}

Our sample includes 360 stars with spectral types from F7 to K4 (spectral types and $B-V$ values were retrieved from the Simbad database at the $\mathrm{CDS}^{1}$ ), observed with HARPS by several groups: Sousa et al. (2008), Ramírez et al. (2014), Marsden et al. (2014), Datson et al. (2014), Borgniet et al. (2017), Lagrange et al. (2013), and Gray et al. (2015). The spectra are available in the ESO archives, and we selected only spectra with an average signal-to-noise ratio above 100 , leading to a total of $19510 \mathrm{spec}-$ tra (i.e., 54 spectra on average per star, a median value of 25 , and a number of spectra per star from 2 to 1230). HARPS spectra cover a [3780-6910] $\AA$ wavelength range, with a resolution of $\sim 120000$. Table 1 lists these surveys and indicates the number of stars of our sample that were extracted from them. We note that many stars have been studied by more than one group (hence the total is larger than 360).

Temperatures are not available for all stars from a single source, therefore we combined several references. As there is usually a systematic offset between these temperature scales

\footnotetext{
1 https://simbad.u-strasbg.fr/simbad/
}

(due to different methods - spectroscopic or photometric -, implementations, models, and data), we computed temperature shifts between pairs of samples (for stars in common) to apply a correction. As a large proportion of our stars are in the sample studied by Sousa et al. (2008), we used their temperature scale as a reference. We then used the following temperatures, in that order depending on availability: Sousa et al. (2008), Ramírez et al. (2014) shifted by $+5 \mathrm{~K}$, Gray et al. (2006) shifted by $+42 \mathrm{~K}$, Marsden et al. (2014) shifted by +13 K, Allende Prieto \& Lambert (1999) shifted by $-41 \mathrm{~K}$, and Holmberg et al. (2009) shifted by $-71 \mathrm{~K}$.

As for temperatures, ages were retrieved from various sources. The differences in age for a given star between different authors present a very large dispersion, which is a significant source of uncertainty on these values. We used the following sources, in that order depending on availability: Holmberg et al. (2009), Delgado Mena et al. (2015), Ramírez et al. (2014), Marsden et al. (2014), and Borgniet (2015).

The activity level is characterized by the usual $\log R_{\mathrm{HK}}^{\prime}$ (chromospheric emission), computed from the analysis of all spectra (see Paper I), as the $\log R_{\mathrm{HK}}^{\prime}$ is strongly related to the presence of activity (e.g., Meunier et al. 2010). It is therefore contemporary to the estimation of the convection amplitude performed in this paper.

Finally, the metallicities $[\mathrm{Fe} / \mathrm{H}]$ were also extracted from different sources. We used the following sources, in this order depending on availability: Sousa et al. (2008), Ramírez et al. (2014), Marsden et al. (2014), Gray et al. (2006), Holmberg et al. (2009), and Delgado Mena et al. (2015).

Figure 1 shows the distribution of these properties for all stars in our sample. The sample is biased toward old, solar-type, and not very active stars. We only included stars with a low $v \sin i$, that is, lower than $5 \mathrm{~km} \mathrm{~s}^{-1}$, as in Paper I. The $v \sin i$ values were mostly retrieved from Nordström et al. (2004), and when not available there, from Jenkins et al. (2011), Valenti \& Fischer (2005), dos Santos et al. (2016), Strassmeier et al. (2000), and Lovis et al. (2005). The reason for this selection is that in contrast to stars with a low $v \sin i$, the differential velocity shift for stars with large $v \sin i$ exhibits a significant decrease, which may be a bias. In principle, the velocity field (inside granules) affects different parts of spectral lines differently because they correspond to different altitudes in the atmosphere. When the $v \sin i$ is large, the contribution from different altitudes is mixed, which could affect the measured radial velocity, and we suggest that the differential velocity we determine (only around line center) is mixed with the contribution from various altitudes.

Table A.1 provides the list of the 360 stars in our sample as well as their properties and references. 

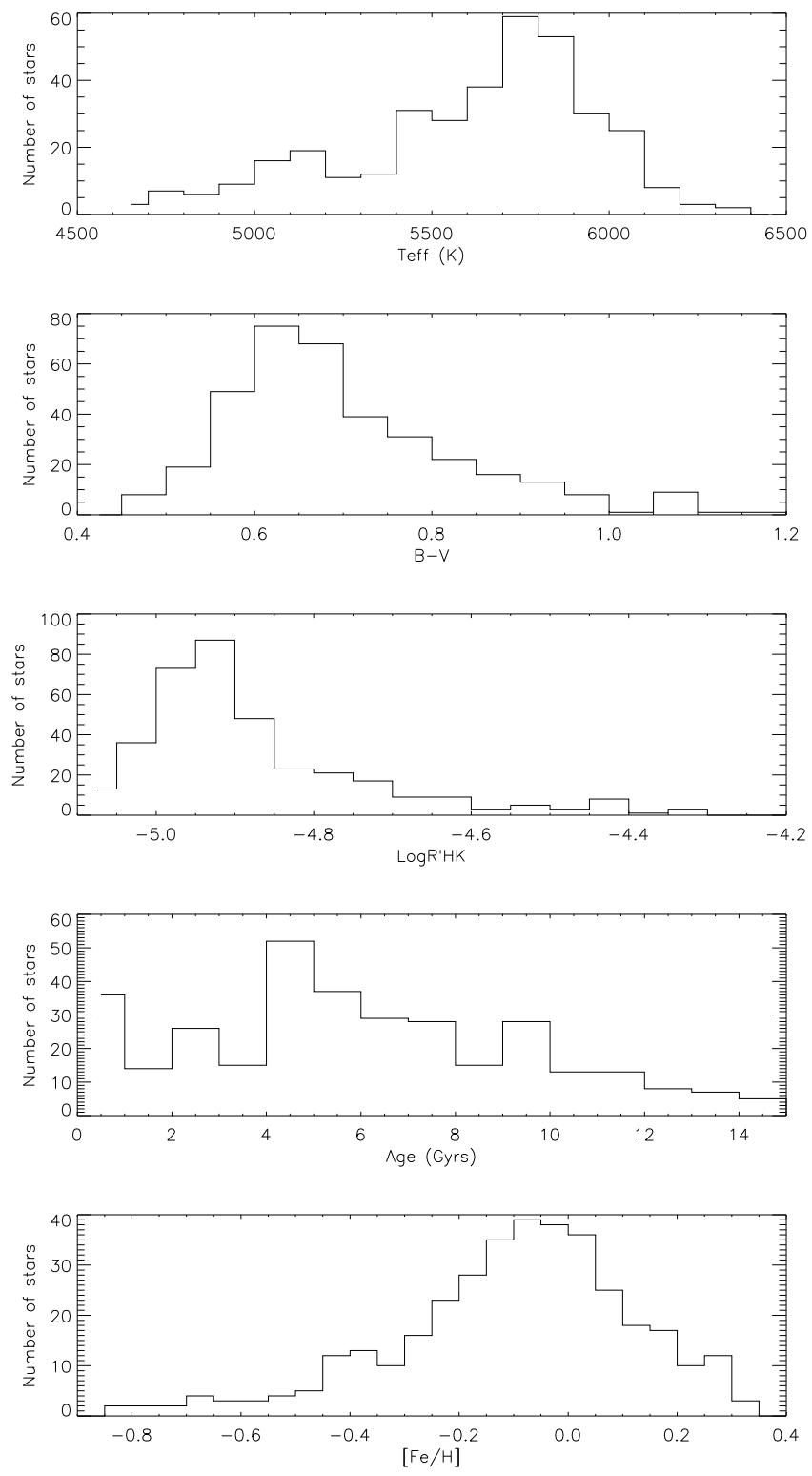

Fig. 1. First panel: histogram of $T_{\mathrm{eff}}$ in our sample. Second panel: same for $B-V$. Third panel: same for $\log R_{\mathrm{HK}}^{\prime}$. Fourth panel: same for ages. Fifth panel: same for $[\mathrm{Fe} / \mathrm{H}]$.

\subsection{Spectral line analysis}

In this section, we briefly describe the analysis performed on the HARPS spectra for all stars in our sample. We refer to Paper I for more details and discussions. We took the following steps:

- The continuum of the spectra is normalized to a flux of 1. A first estimation is made by determining the upper envelope, and it is followed by a correction by a factor taking into account the noise. The full procedure is detailed in Paper I.

- Spectral lines corresponding to TiI and FeII from Dravins (2008) and FeI from Nave et al. (1994) are selected within the range 5000-6400 $\AA$. The position of the line is derived from a fit around line center for each of these lines on all individual spectra, and the velocity shift is computed from the difference between this wavelength and the laboratory wavelength. A total of 237 lines is used. We note

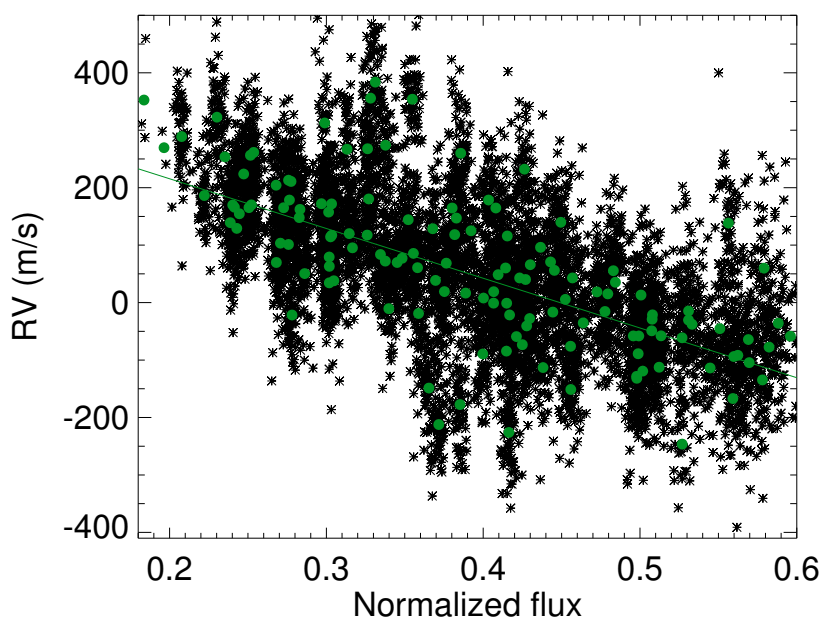

Fig. 2. RV versus a normalized flux at the bottom of the lines for HD 223171 (G2). Stars represent individual measurements, while the green dots correspond to the temporal average for each spectral line. The straight line is a linear fit on the green dots (from Meunier et al. 2017).

that the convective blueshift is expected to be slightly higher (Dravins et al. 1986) for FeII lines than for FeI lines: we obtain convective blueshifts that remain within the observed dispersion for the TiI and FeI lines, and there are in fact very few FeII lines in our sample so that in practice our results is dominated by the TiI and FeI lines (as shown in the line list in Paper I).

- For each star and each observing time, we compute the averaged RV (i.e., over all spectral lines). This average is then subtracted from each individual velocity shift at that time step. We then average over time all velocities for a given spectral line. The resulting RV versus line flux for that star (one per spectral line) is then fit with a linear function.

- The slope of the linear fit measures the amplitude of the differential blueshift, which we named the TSS (for "third signature slope", following the third signature proposed by Gray 2009), in $\mathrm{m} / \mathrm{s} /(\mathrm{F} / \mathrm{Fc}$ ) (with $\mathrm{F} / \mathrm{Fc}$ representing the intensity after normalization by the continuum $\mathrm{Fc}$ ). We note that the unit $\mathrm{m} / \mathrm{s}$ is used in the following to simplify the notations. Individual and temporally averaged measurements are illustrated in Fig. 2.

- The TSS is used as a criterion to characterize the amplitude of the convective blueshift (i.e., the absolute blueshift) after a normalization with the solar values, based on the assumption made by Gray (2009) that the shape of the differential shift of spectral lines is representative of the absolute convective blueshift: $\mathrm{RV}_{\text {convbl }}=\mathrm{TSS} \times \mathrm{RV}_{\text {convblø }} / \mathrm{TSS}_{\odot}$. We normalized it to the solar values computed in Paper I: solar TSS of $-776 \mathrm{~m} / \mathrm{s}$, which we derived from the solar spectrum of Kurucz et al. (1984) degraded to the HARPS spectral resolution; solar convective blueshift of $355 \mathrm{~m} / \mathrm{s}$, which we computed from the absolute solar RV versus spectral line depths of Reiners et al. (2016) for lines identified in the solar spectra of Kurucz et al. (1984) and assuming that the RV computed from cross-correlations between spectra will give more weight to deep lines. We recall that the derived absolute convective blueshift, either the solar value of $355 \mathrm{~m} / \mathrm{s}$ or the stellar values inferred from the above relation, therefore 
correspond to this set of lines and procedure, and care must be taken when comparing with other works.

In addition, we computed the $\log R_{\mathrm{HK}}^{\prime}$ from each spectrum. We use the average value for each star in Sect. 3 and consider its variability in Sect. 4. The RVs corresponding to our spectra computed by the Data Reduction Software (DRS) at ESO were also retrieved from the archive data to compare the two variabilities in Sect. 4. For some of these stars, we performed a correction when the variability was dominated by a binary component or a known planet.

\section{Differential velocity analysis}

\section{1. $T_{\text {eff }}, B-V$, and activity relationship}

The first panel of Fig. 3 shows $B-V$ versus $T_{\text {eff }}$ : there is a very good correlation, as expected, but we point out here the low dispersion in this relationship, to be compared to the other plots. The second and third panels show the TSS versus $B-V$ and $T_{\text {eff }}$, respectively. We observe a very good relation as well, with a dispersion that is not much larger than the $B-V$ versus $T_{\text {eff }}$ relation, and very few outliers. As pointed out in Sect. 2.1, there is a large uncertainty on the $T_{\text {eff }}$ values: the third panel shows the range covered by $T_{\text {eff }}$ from the different references indicated in Sect. 2.1 (we show it only in this panel for clarity). Finally, the last two panels show a reconstruction of the convective blueshift versus $T_{\text {eff }}$ and $B-V$, respectively, for our sample of 360 stars following the method described in Sect. 2.2. We do not see any saturation toward high $T_{\text {eff }}$ (or low $B-V$ ), as was marginally visible in Paper I. On the other hand, there is a clear saturation at low $T_{\text {eff }}\left(T_{\text {eff }}\right.$ below $\left.5000 \mathrm{~K}\right)$, with a convective blueshift around $100 \mathrm{~m} / \mathrm{s}$. As in Paper I, these convective blueshifts are higher than those derived from the numerical simulation made by Allende Prieto et al. (2013). Such a comparison is made after taking into account the difference in convective blueshift computed for our set of lines and the small wavelength range used by Allende Prieto et al. (2013) to simulate Gaia observations (i.e., 8470-8740 ̊). The spectral resolution and method to infer the wavelength shift are different, however, so that Allende Prieto et al. (2013) may have underestimated the effect as they used a cross-correlation between the spectra and a template spectrum to estimate the velocity shifts: a more precise analysis of simulated spectra should therefore be performed to understand the origin of the difference better.

Figure 4 shows the individual TSS (one average per star) versus $\log R_{\mathrm{HK}}^{\prime}$ for ten $T_{\text {eff }}$ ranges covering the full available range. There is a good correlation between the two variables for almost all bins. A few correlations are not well defined, especially when combining a small sample (at very high or very low $T_{\text {eff }}$ ) and a low amplitude of the TSS (at low $T_{\text {eff }}$ ). The bin $5516-5685 \mathrm{~K}$ also lacks of stars at high activity levels.

Finally, Fig. 5 shows the TSS averaged in each of these $T_{\text {eff }}$ bins versus $T_{\text {eff }}$, as well as the slope of the TSS versus $\log R_{\mathrm{HK}}^{\prime}$ and the ratio of the two. The last panel shows the number of stars in each bin, with a strong bias toward solar-type stars: some of the bins are not well sampled. The ratio between the slope and the average TSS is very interesting because it characterizes the attenuation of the convective blueshift that is due to activity for a given variation of the activity level. The average is equal to 1.47 (1.27 when weighted by the inverse of the uncertainties to the power of two), with a $1 \sigma$ uncertainty of 0.18 . Although there is no global trend over the full range, there is a trend for $T_{\text {eff }}$ higher than $5200 \mathrm{~K}$, and the ratio can be modeled by $-2.64+6.77$
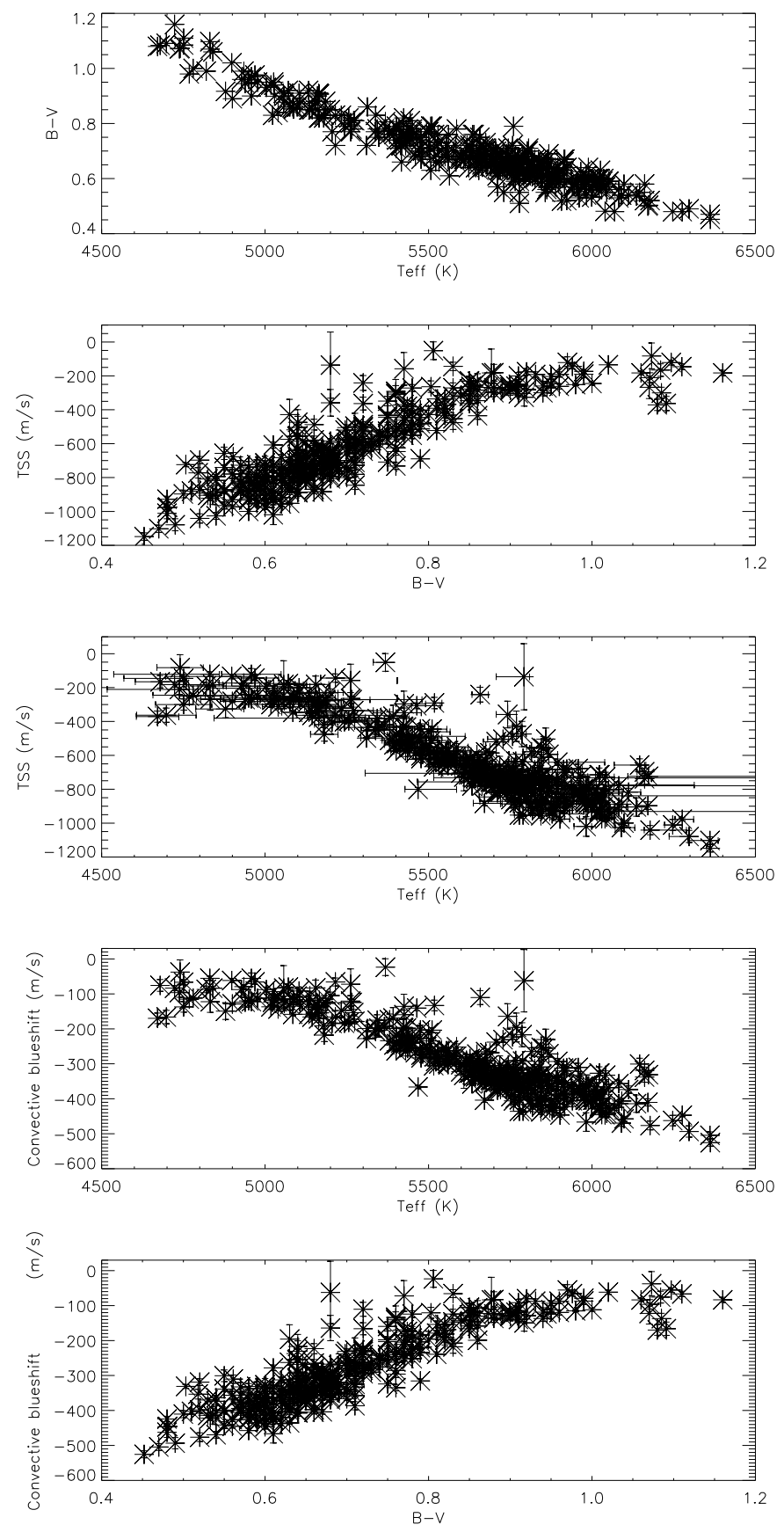

Fig. 3. First panel: $B-V$ versus $T_{\text {eff }}$ for the 360 stars in our sample. Second panel: same for the TSS versus $B-V$. Third panel: same for the

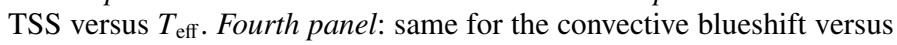
$T_{\text {eff. }}$. Fifth panel: same for the convective blueshift versus $B-V$.

$10^{-4} \times T_{\text {eff }}$ : more massive stars tend to have a higher ratio than less massive stars. At a $T_{\text {eff }}$ of $6300 \mathrm{~K}$, the attenuation should therefore be about $35 \%$ stronger than for the Sun, while at a $T_{\text {eff }}$ of $5200 \mathrm{~K}$, the attenuation factor should be $25 \%$ weaker than for the Sun. This means that the more massive stars, which also have a stronger convective blueshift, should exhibit even larger RV variations because of the attenuation of the convective blueshift in plages since the ratio is higher than for the Sun.

These observations on a much larger sample therefore confirm the results of Paper I, especially the global trend versus $T_{\text {eff }}$ and the dependence on activity, and extend the results to a wider range in $T_{\text {eff }}$. The attenuation factor seems to exhibit a trend 
N. Meunier et al.: Variability in stellar granulation and convective blueshift
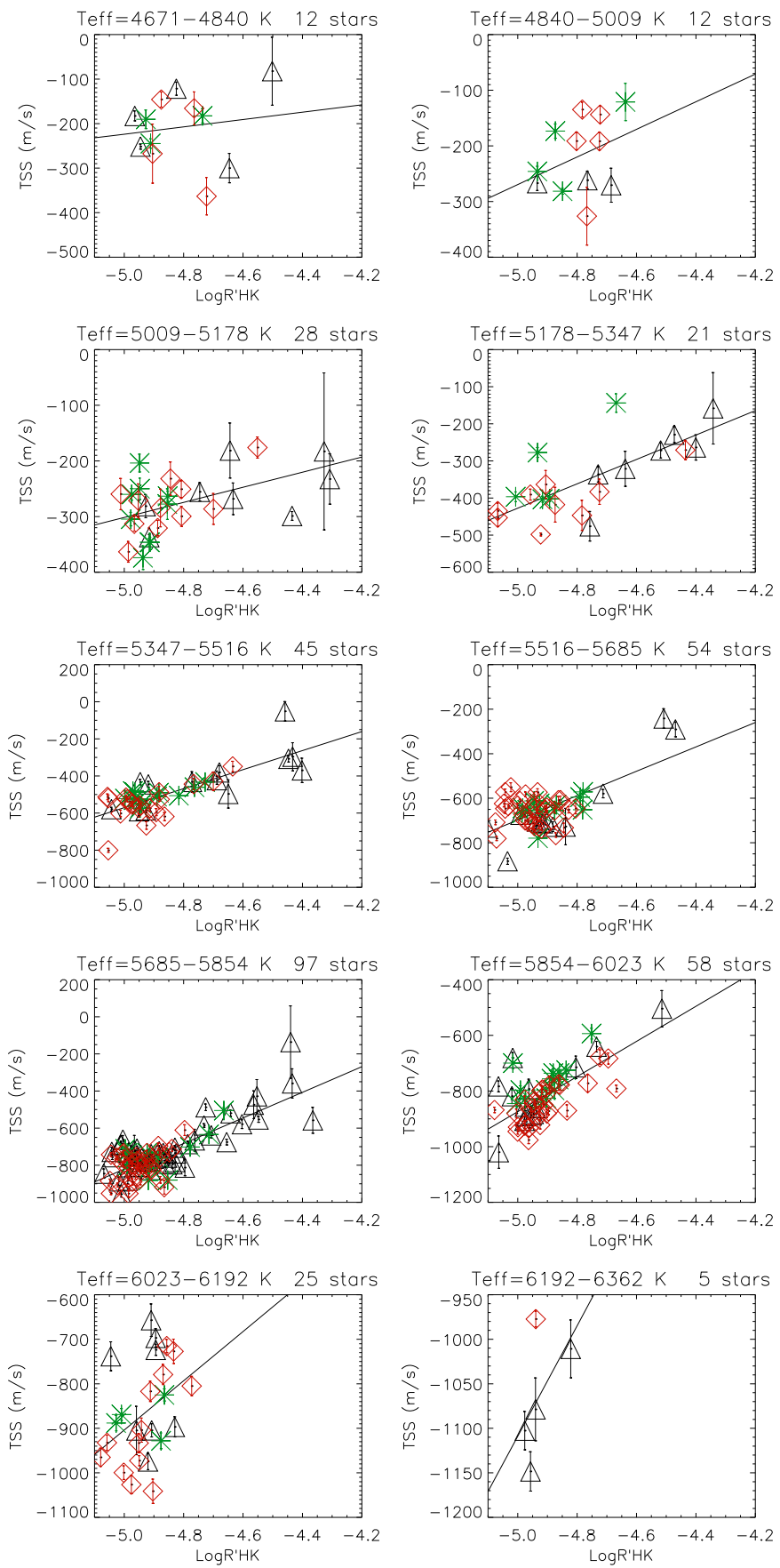

Fig. 4. TSS versus $\log R_{\mathrm{HK}}^{\prime}$ for ten $T_{\text {eff }}$ ranges. The solid line is a linear fit. Green stars are stars with an identified activity cycle, red diamond show stars with no cycle, and black triangles represent stars with undetermined behavior.

above $5200 \mathrm{~K}$, which could not be detected from the smaller sample of Paper I.

\subsection{Effect of cyclic activity on the convection-activity relationship}

Figure 6 shows the TSS versus $\log R_{\mathrm{HK}}^{\prime}$ for a selection of stars with $T_{\text {eff }}$ in the range $5662-5918 \mathrm{~K}$, selected to be similar to the range covered by the sample of Marsden et al. (2014), who focused on solar-type stars. We identify 21 stars in this plot for which Lovis et al. (2011) identified cyclic activity (green) and 55 stars for which they identified strong variability, but no cycle
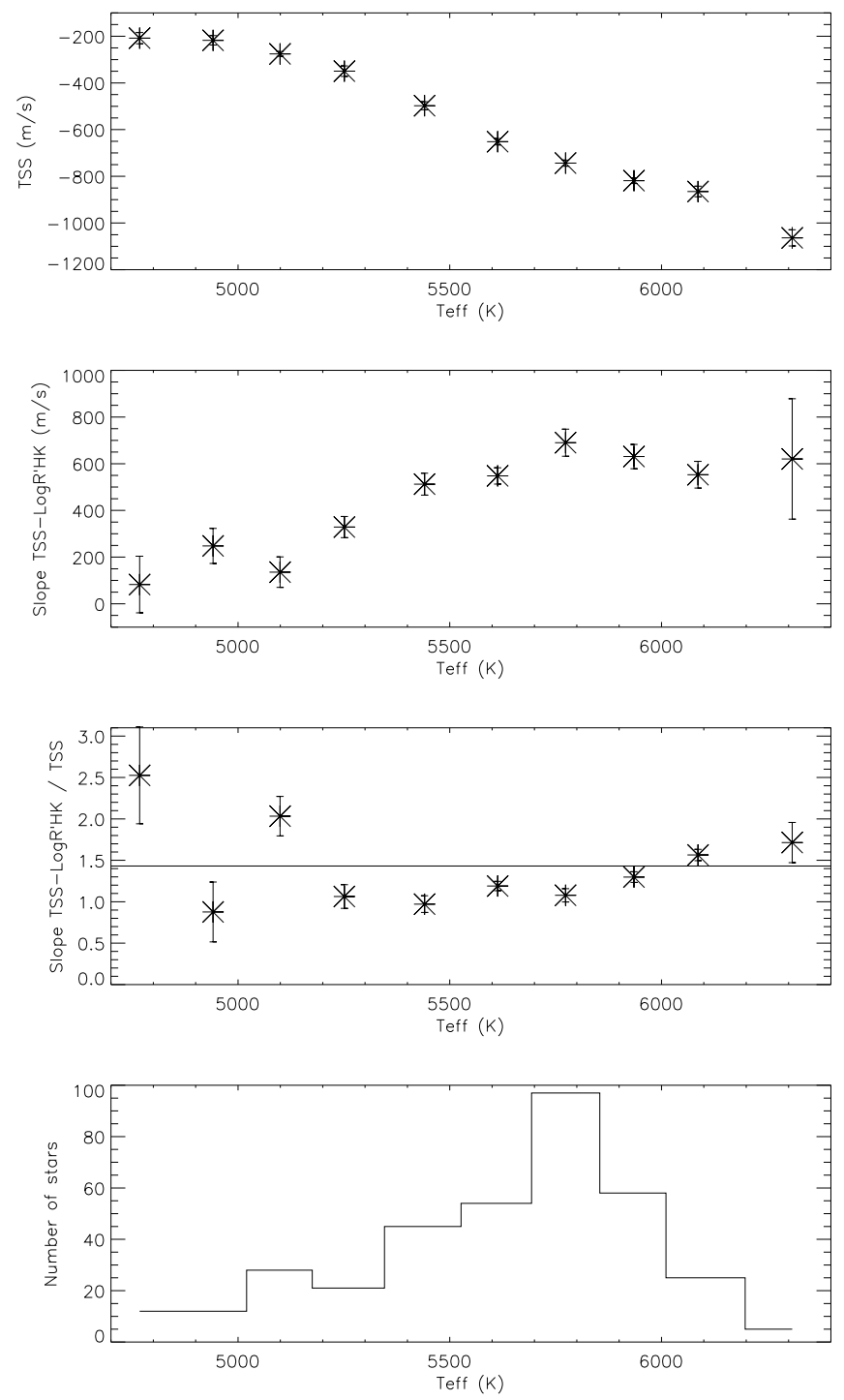

Fig. 5. First panel: average TSS versus $T_{\text {eff }}$ in ten $T_{\text {eff }}$ bins. Second panel: same for the slope of the TSS versus Log $R_{\mathrm{HK}}^{\prime}$. Third panel: same for the ratio between the slope and the average. Fourth panel: number of stars in each bin.

(red). We find that these two populations do show a very similar behavior, within $2 \sigma$. It is a small sample, however, especially for stars with a cycle, therefore there is a large uncertainty on the slopes. We note that for many stars in this $T_{\text {eff }}$ range, it is unclear whether they had a cycle, either because they were not studied by Lovis et al. (2011) or because of poor sampling, which prevented such a characterization. A similar distinction in Fig. 4 leads to a similar conclusion.

We observe in Fig. 6 a relatively large dispersion in TSS at low $\log R_{\mathrm{HK}}^{\prime}$, with a deviation from the linear relationship toward a lower convection level. For $\log R_{\mathrm{HK}}^{\prime}$ below -4.95 , we have separated the sample (for $T_{\text {eff }}$ in the range $5662-5918 \mathrm{~K}$ ) into two subsets: stars with a TSS above $1000 \times \log R_{\mathrm{HK}}^{\prime}$ $+4200 \mathrm{~m} / \mathrm{s}$ (solid line in Fig. 6), and stars with a TSS below this line. These two subsets do not show any significant difference in $T_{\text {eff }}, v \sin i$, or age. However, the first set has an average $[\mathrm{Fe} / \mathrm{H}]$ of $0.13 \pm 0.02$ and the other $-0.09 \pm 0.02$ : this could therefore be due to a metallicity effect, which is studied in more detail in Sect. 3.4. 


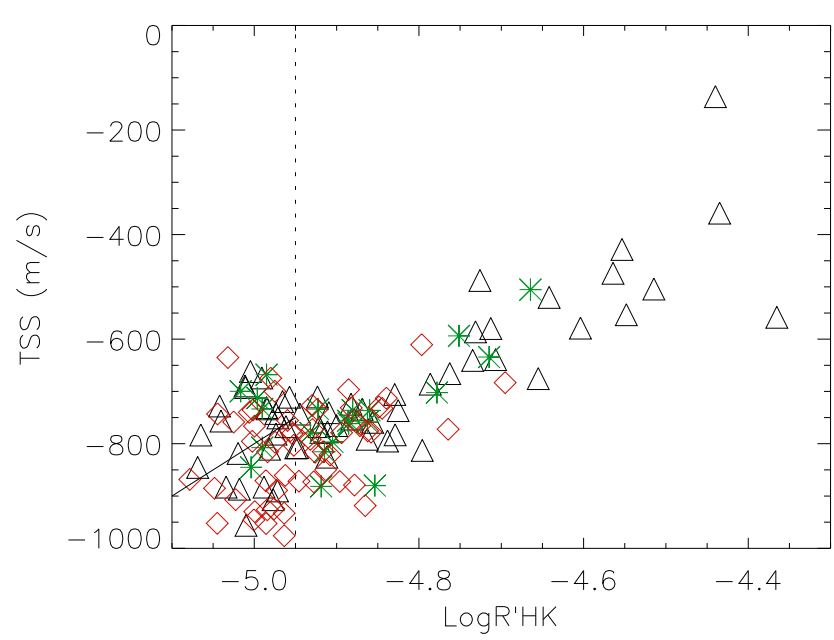

Fig. 6. TSS versus $\log R_{\mathrm{HK}}^{\prime}$ for the 137 stars in our sample with $T_{\text {eff }}$ between 5662 and $5918 \mathrm{~K}$. The color code is similar to that in Fig. 3 The solid line indicates the separation between the stars following the linear behavior and those with a lower convection level in Sect. 3.4, for stars with $\log R_{\mathrm{HK}}^{\prime}$ lower than -4.95 (dotted line).
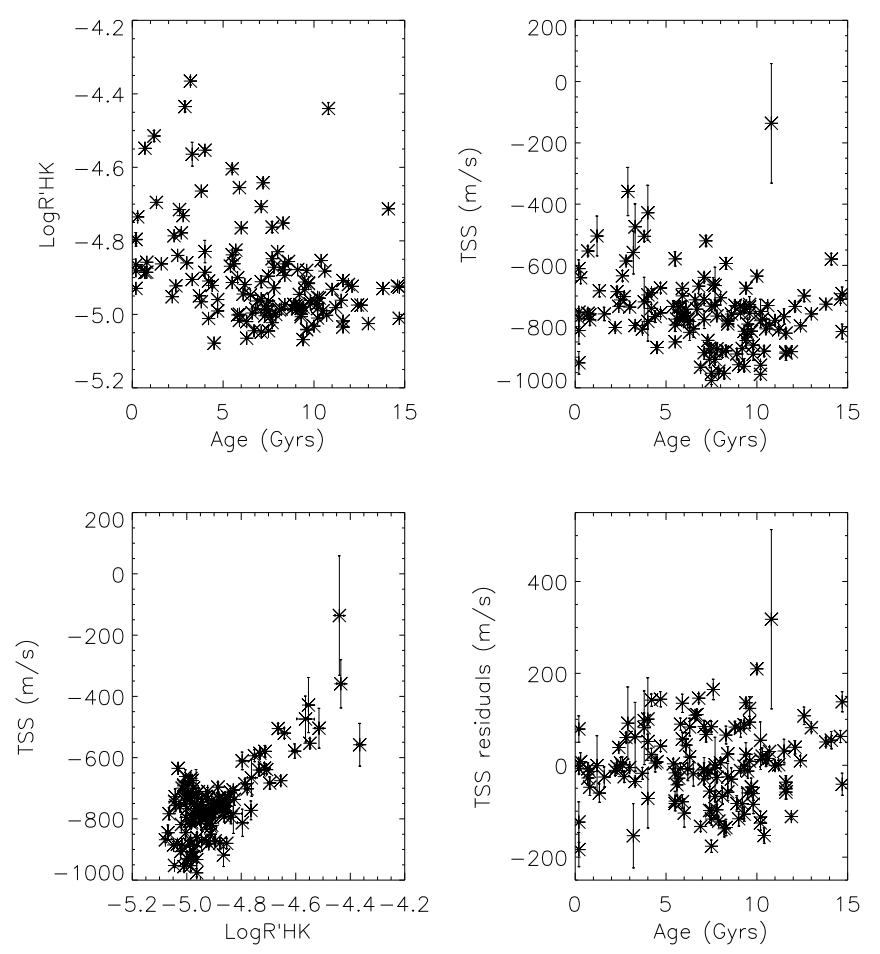

Fig. 7. Upper left panel: $\log R_{\mathrm{HK}}^{\prime}$ versus age for stars with $T_{\mathrm{eff}}$ in the range 5662-5918 K. Upper right panel: same for the TSS versus age. Lower left panel: same for the TSS versus the $\log R_{\mathrm{HK}}^{\prime}$. Lower right panel: same for the residual TSS (after correction from the $\log R_{\mathrm{HK}}^{\prime}$ trend) versus age.

\subsection{Effect of age on the convection-activity relationship}

We now consider stars in the same temperature range, for which we have a relatively large homogeneous sample, and analyze the relationship between TSS and age. Ages are not identified for a few of these 137 stars, so we consider 133 stars here. Figure 7 shows the $\log R_{\mathrm{HK}}^{\prime}$ and the TSS versus age, showing very similar behaviors. After correcting for the strong TSS-Log $R_{\mathrm{HK}}^{\prime}$ dependence (shown in the lower left panel), the residuals versus age do not show any trend and are of much smaller amplitude. The age of the star therefore does not significantly affect the TSS, and it plays a role only through the relationship between age and activity, which is the main factor.

We also separated our sample into two subsamples: 13 stars with an age below $2 \mathrm{Gyr}$, and 120 older stars. We do not observe any difference in average TSS or in slope TSS versus $\log R_{\mathrm{HK}}^{\prime}$, within $1 \sigma$. The small sample of young stars limits this analysis. When considering an age limit of $1 \mathrm{Gyr}$, the sample of young stars is limited to 10 stars and the difference is even less significant.

We conclude that we do not see any significant difference in the behavior between the young and old stars in our sample, and estimate that plages, which are more frequently present in young stars (with a large $\log R_{\mathrm{HK}}^{\prime}$ than in older stars) may have similar properties as plages in older stars.

We note that the separation between young and old stars here refers to their activity level. The age limit corresponding to the spot-dominated regime (young stars) and plagedominated regime (old stars) in Lockwood et al. (2007) is not well defined, but our 2 Gyr threshold is consistent with the limit between spot-dominated and plage-dominated regimes in Lockwood et al. $(2007)^{2}$.

We also recall that our young star subsample is biased toward slowly rotating stars because it is difficult to measure the TSS for stars with a $v \sin i$ higher than $5 \mathrm{~km} \mathrm{~s}^{-1}$. We therefore cannot exclude that plages in fast-rotating stars could have different properties. It may also be a bias toward stars seen pole-on.

\subsection{Effect of metallicity on the convection-activity relationship}

We have shown in Sect. 3.2 that for stars in the 5662-5918 K range and $\log R_{\mathrm{HK}}^{\prime}$ below -4.95 , there is a strong link between the TSS and $[\mathrm{Fe} / \mathrm{H}]$, the larger $[\mathrm{Fe} / \mathrm{H}]$ being associated with a lower convective blueshift. We now investigate this effect in more detail. The upper panel of Fig. 8 shows separately the TSS versus $\log R_{\mathrm{HK}}^{\prime}$ for $T_{\mathrm{eff}}$ in the same range, but for different metallicities. The high-metallicity stars, showing the deviation toward a lower convective blueshift, also correspond to $\operatorname{low} \log R_{\mathrm{HK}}^{\prime}$ and a lower convective blueshift than the low-metallicity stars. Furthermore, we also observe that there is a relationship between the metallicity and the $\log R_{\mathrm{HK}}^{\prime}$, as shown in the last panel: this plot is very similar to the results obtained by Jenkins et al. (2008): for $\log R_{\mathrm{HK}}^{\prime}$ smaller than -4.80 , low metallicity is associated with more active stars. The origin of this effect is not discussed by Jenkins et al. (2008), but it could be either due to the effect of metallicity on the estimation of the activity level using the $\log R_{\mathrm{HK}}^{\prime}$ itself (the computation of which does not include the effect of metallicity, see Rocha-Pinto \& Maciel 1998; Wright 2004; Judge \& Saar 2007; Mittag et al. 2013), or it could be due to the fact that stars with a low metallicity are more active, for example because they have a more vigourous small-scale convection (although we found no suggestion of this possibility in the literature), leading to a higher convective blueshift.

2 The Hyades isochrone at 625 Myr (Perryman et al. 1998) falls well within the young star regions. The limit between the two regimes corresponds to older stars and seems to exhibit a decreasing $\log R_{\mathrm{HK}}^{\prime}$ as $B-V$ increases, while we expect the isochrones to have the opposite behavior (e.g., Mamajek \& Hillenbrand 2008): the age limit derived from the isochrones of (e.g., Mamajek \& Hillenbrand 2008) would then be close to $1 \mathrm{Gyr}$ for $B-V$ around $0.5,2 \mathrm{Gyr}$ around solar mass stars, and $3 \mathrm{Gyr}$ for $B-V$ around 1 . 

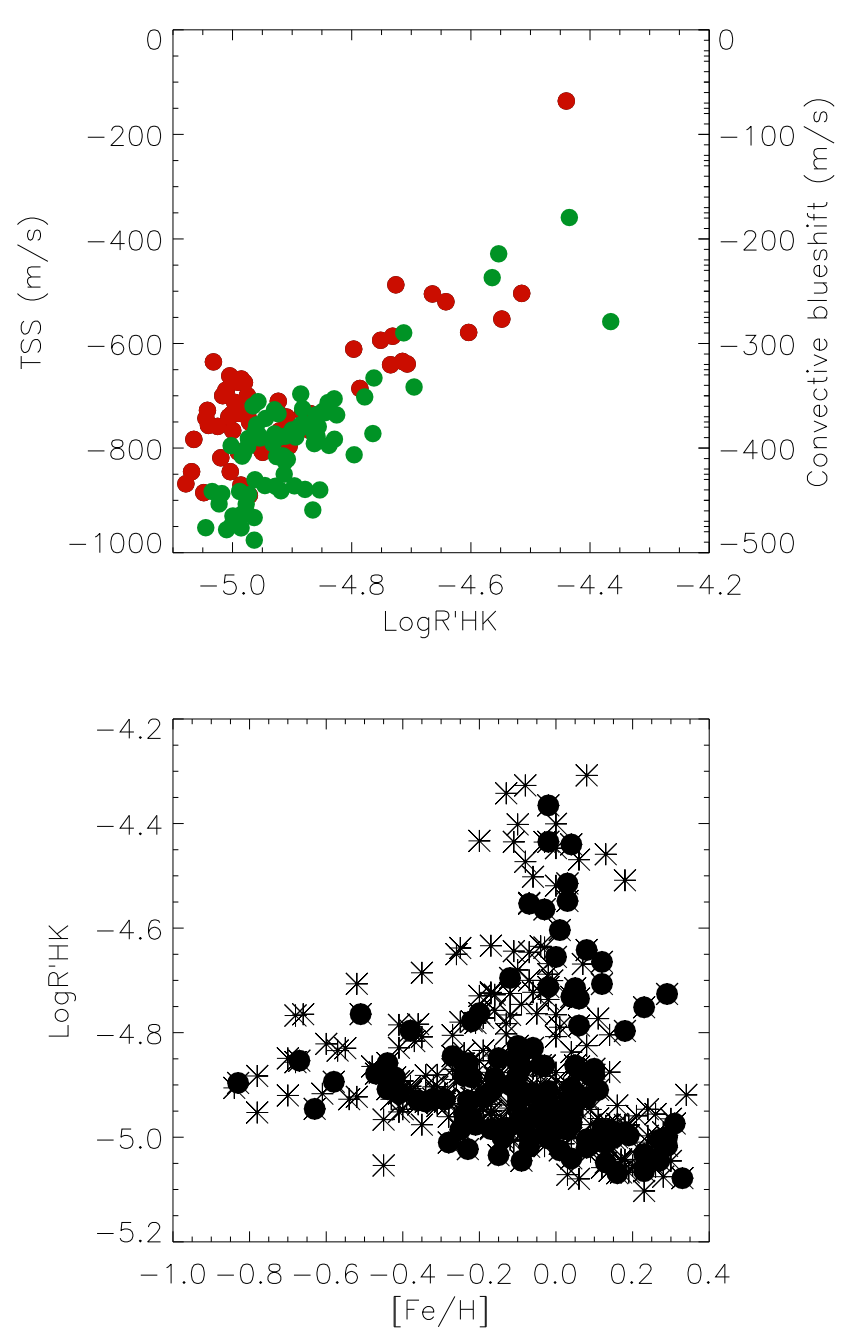

Fig. 8. Upper panel: TSS (left axis) and convective blueshift (right axis) versus $\log R_{\mathrm{HK}}^{\prime}$ for $T_{\text {eff }}$ between 5662 and $5918 \mathrm{~K}$, for stars with a positive $[\mathrm{Fe} / \mathrm{H}](\mathrm{red})$ and with a negative $[\mathrm{Fe} / \mathrm{H}]$ (green). Lower panel: $\log R_{\mathrm{HK}}^{\prime}$ versus $[\mathrm{Fe} / \mathrm{H}]$ for stars with $T_{\text {eff }}$ between 5662 and $5918 \mathrm{~K}$ (circles) and the other stars of our sample (stars).

Our interpretation of these plots is the following: we observe two competing effects here. On one hand, low metallicity seems to be associated with a higher convective blueshift. On the other hand, it is also associated with higher activity levels, hence a reduced convective blueshift. This can explain the position of the high-metallicity stars in Fig. 8, with a lower |TSS| than would be expected from the linear law.

We have quantified this difference in Appendix B and found that for a $\Delta[\mathrm{Fe} / \mathrm{H}]$ of 0.32 , the convective blueshift is indeed 10 $12 \%$ lower (in absolute value) for stars with the highest metallicity compared to those with the lowest.

Several groups have studied the effect of metallicity on convection using hydrodynamical simulations of convection in various types of stars, with complex effects. Allende Prieto et al. (2013) found more vigourous convection for low metallicities (as observed here), although to a lesser degree: for $T_{\text {eff }}=5700 \mathrm{~K}$ and $\log g=4.5$, the difference in velocity shift (with the factor used in this paper) between a $\Delta[\mathrm{Fe} / \mathrm{H}]$ of 0.27 is $4.4 \mathrm{~m} / \mathrm{s}$, which represents $2.4 \%$ of the low-metallicity value, that is, it is 4-6 times lower than our estimation. Magic et al. (2013) also found a higher granulation contrast for low-metallicity stars (around 25\%), but surprisingly, this did not seem to produce a significant difference in velocity shift for the $T_{\text {eff }}$ corresponding to our sample (Magic et al. 2014). Tremblay et al. (2013) also found a higher granulation contrast at low metallicity, but with a smaller effect of metallicity than Magic et al. (2013). Therefore, although simulations are qualitatively in agreement with our observations (low metallicity corresponding to a more vigourous small-scale convection), the observed effect of metallicity seems to be significantly larger than what was obtained in numerical simulations.

Metallicity is important because some results have indicated that stars hosting giant planets may be more metallic on average than the Sun, leading to some conclusion on formation processes (e.g., Santos et al. 2003; Fischer \& Valenti 2005). Exoplanet surveys are usually biased toward less active stars (e.g., Lovis et al. 2011), however, to limit the effect of activity on RV, based on $\log R_{\mathrm{HK}}^{\prime}$ values, but if low $\log R_{\mathrm{HK}}^{\prime}$ values are associated with higher metallicities, as shown by Jenkins et al. (2008), then this could alter the metallicity-exoplanet relationship. This may explain why such a relationship is not observed in transit surveys (Fridlund et al. 2010).

\section{Relationship between activity and RV variations}

\subsection{Statistical analysis of the sample}

Meunier et al. (2010) have shown that long-term RV variations should be dominated by the inhibition of the convective blueshift in solar-type stars, if they behave like the Sun. There are indications of correlations between RV amplitudes and $\log R_{\mathrm{HK}}^{\prime}$ amplitudes, as shown by the trend of the RV-Log $R_{\mathrm{HK}}^{\prime}$ slope versus $T_{\text {eff }}$ obtained by Lovis et al. (2011). Isaacson \& Fischer (2010) also estimated the RV jitter versus average activity level for different stellar types, but the derived lower envelope as a function of spectral type is not well constrained. In Paper I, in addition to characterizing the convective blueshift-activity relationship, we have also estimated the amplitude in RV compared to the amplitude in $\log R_{\mathrm{HK}}^{\prime}$ for a small sample of stars and observed a trend, with a large dispersion. Overall, it is not clear up to which point solar-type stars behave like the Sun as obtained by Meunier et al. (2010). With a larger sample such as studied in this paper, we now have the opportunity to estimate the proportion of stars that follows what we call the "solar pattern" (RV long-term variations correlated with activity variability due to the inhibition of the convective blueshift, with a possible modulation of the effect with inclination, angle between the rotation axis, and the line of sight, and including small variability for both RV and $\log R_{\mathrm{HK}}^{\prime}$ ), and those that do not, keeping in mind possible biases in our sample. We therefore now characterize the stars in our sample, following the characteristics described in Table 2.

We first eliminate some stars from this statistical analysis for several reasons, mostly because they do not have enough points to characterize any long-term variations, and a few because the RV time series from the ESO DRS were not reliable. We also counted 26 stars (category 9 in Table 2) separately with known sources of RV variations that we did not correct for: 4 binary stars, 2 variable stars, and 20 stars with a known planetary signal that we did not correct.

We identified 165 stars that follow the solar pattern: their $\mathrm{RV}$ and $\log R_{\mathrm{HK}}^{\prime}$ are correlated, or if they are not, both exhibit a small variability. We also included the stars with large $\log R_{\mathrm{HK}}^{\prime}$ variation but small RV variation, which could be due to a low convection level or inclination effects Borgniet et al. (2015), or both. These 165 stars are categorized into five groups (categories 1-5) in Table 2. 
Table 2. Statistics of the RV-Log $R_{\mathrm{HK}}^{\prime}$ relationship.

\begin{tabular}{llll}
\hline \hline Categ. & Definition & NB & $\begin{array}{l}\text { Perc. } \\
(\%)\end{array}$ \\
\hline 1 & Good sampling, excellent correlation & 13 & 7.9 \\
2 & Moderate sampling, good correlation & 48 & 29.0 \\
3 & Weak correlation & 15 & 9.1 \\
4 & Weak signals, no correlation & 70 & 42.4 \\
5 & Strong Log $R_{\mathrm{HK}}^{\prime}$, weak RV & 19 & 11.5 \\
$1-5$ & & 165 & - \\
\hline 6 & Strong signals, no correlation & 12 & 28.6 \\
7 & Strong signals, anticorrelation & 11 & 26.2 \\
8 & Strong RV, weak Log $R_{\mathrm{HK}}^{\prime}$ & 19 & 45.2 \\
$6-8$ & & 42 & - \\
\hline 9 & Strong RV, identified origin & 26 & - \\
\hline
\end{tabular}

Notes. Number of stars in each category NB, for the selected stars (207). The percentages indicate the fraction with respect to 148 for categories $1-5$ and 42 for categories $6-8$. Category 9 corresponds to a strong RV signal with a known origin (mostly presence of planets). Correlation means between $\mathrm{RV}$ and $\log R_{\mathrm{HK}}^{\prime}$ time series; weak-strong signals are related to the amplitudes of $\mathrm{RV}$ and $\log R_{\mathrm{HK}}^{\prime}$, especially on long or median timescales, and not to the average level.

A second group of 42 stars were identified and did not follow this solar pattern, as they exhibit large RV variations that cannot be explained by their $\log R_{\mathrm{HK}}^{\prime}$ variations: we observed either no correlation, an anticorrelation, or a weak $\log R_{\mathrm{HK}}^{\prime}$ variation (categories 6 to 8). This means that for these stars the RV variations are dominated by another unknown effect. When we add the 26 stars mentioned above whose RV variations are dominated by a known effect other than the convection inhibition, this represents $29 \%$ of this sample of 233 stars (18\% with unknown origin, $11 \%$ with a known origin), while those following the pattern represent $71 \%$.

The definition of the categories is such that there is a certain continuum between them, which introduces an uncertainty, probably on the order of a few percent. We also recall that the sample is biased toward solar-type stars, for which we have shown that the inhibition of the convective blueshift is important, so we may underestimate category 7 (unless lower mass stars have a larger variability, which can be observed from the analysis of the cycle amplitudes as a function of the average activity level in Lovis et al. 2011). On the other hand, it is also biased toward stars that are not very active (most are less variable than the Sun), therefore we may underestimate categories 1,2 , and 8 . In this small sample we do not observe any significant difference between the number of stars in the different categories for low- and high-mass stars, however.

Finally, we examined the distribution of young stars in these categories. The results are summarized in Table 3 . Young stars seem to be overrepresented in categories $6-8$ or $6-9$, which is significant at the $1 \sigma$ level.

\section{2. $R V$ variability}

We now focus on stars that follow the convection inhibition pattern, that is, stars in categories 1-5. As in Paper I, we selected stars with enough points to estimate a reasonably representative long-term amplitude and average the values in bins of at least five points. We identified the observing times corresponding to the minimum and maximum $\log R_{\mathrm{HK}}^{\prime}$, for these two observing times we computed $\Delta \log R_{\mathrm{HK}}^{\prime}$ and $\Delta \mathrm{RV}$. This led to a selection
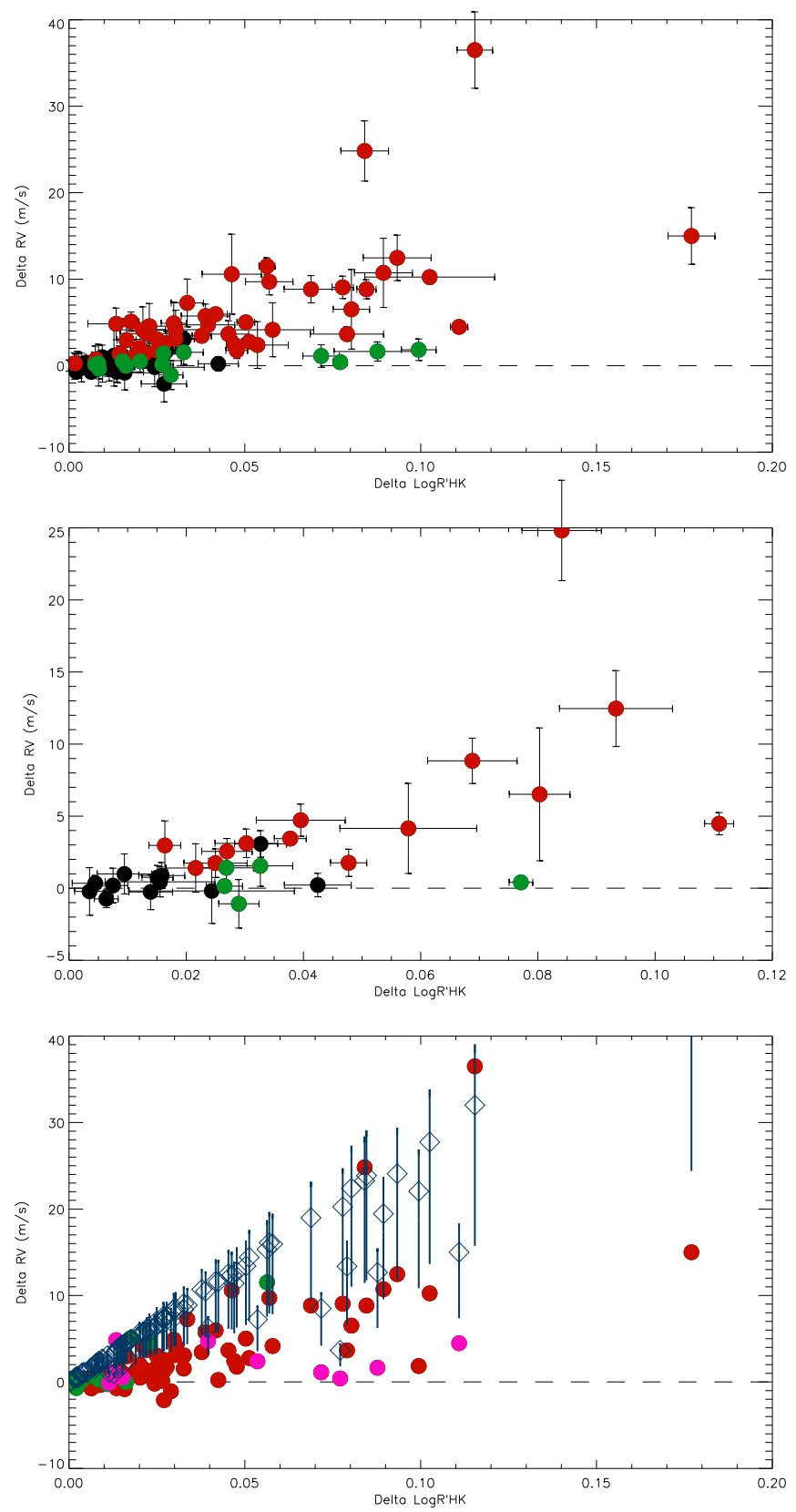

Fig. 9. Upper panel: $\Delta \mathrm{RV}$ versus $\Delta \log R_{\mathrm{HK}}^{\prime}$ for our selection of 106 stars, from categories 1-3 (red), category 5 (green), and category 4 (black). Middle panel: same for the 31 stars with $\Delta t$ larger than 1000 days. Lower panel: same as the upper panel, but showing the different spectral types: F stars (green circles), G stars (red circles), and $\mathrm{K}$ stars (pink circles). The blue diamonds indicate the reconstructed RV amplitude, and each vertical blue line shows the expected effect of inclination.

of 106 stars. We introduced an additional selection on the time range $\Delta t$ separating the two observing times identified as minimum and maximum: we then considered a second subsample of stars for which $\Delta t$ is larger than 1000 days as well (to focus on long-term variations), which led to a sample of 31 stars.

The results are shown in Fig. 9 for these two selections of 106 and 31 stars. We do observe a trend with larger $\Delta \log R_{\mathrm{HK}}^{\prime}$ for larger $\Delta \mathrm{RV}$. As in Paper I, there is a large dispersion, which was discussed as being due not only to the expected difference in response for different spectral types, but also to inclination 
Table 3. Young star categories.

\begin{tabular}{llll}
\hline \hline Categ. & $\begin{array}{l}\text { NB } \\
\text { young stars }\end{array}$ & $\begin{array}{l}\text { NB } \\
\text { total }\end{array}$ & $\begin{array}{l}\text { Fraction of } \\
\text { young stars }\end{array}$ \\
\hline $1-5$ & 13 & 165 & $0.079_{-0.022}^{+0.030}$ \\
$6-8$ & 13 & 42 & $0.309_{-0.116}^{+0.158}$ \\
9 & 4 & 26 & $0.154_{-0.089}^{+0.133}$ \\
$6-9$ & 19 & 68 & $0.279_{-0.087}^{+0.111}$ \\
\hline
\end{tabular}

Notes. Categories are defined in Table 2. Young stars are those younger than 2 Gyr.

effects that cannot be removed. We also note that although the sample has been improved, there is a lack of stars with a large $\Delta \log R_{\mathrm{HK}}^{\prime}$ : there are almost no stars in our sample with an amplitude larger than solar (0.1 at least), which is a problem.

We note a few outliers with $\Delta \mathrm{RV}$ between -10 and $-5 \mathrm{~m} / \mathrm{s}$. This is usually within the noise at the $3 \sigma$ level and could be due to particular sampling effects. We also recall that there is a certain continuity between the categories, so these few stars could be close to categories 6-9.

As in Paper I, we also estimated for each star the RV amplitude $\Delta \mathrm{RV}_{\text {conv }}$ that is expected given their observed $\Delta \log R_{\mathrm{HK}}^{\prime}$, assuming they follow the laws observed in Sect. 3 and that the RV amplitude is only due to the inhibition of the convective blueshift (see Paper I for more details):

$\Delta \mathrm{RV}_{\text {conv }}=G \times \mathrm{RV}_{\text {convbl } \odot} / \mathrm{TSS}_{\odot} \Delta \log R_{\mathrm{HK}}^{\prime}$,

where $G$ is the slope of the TSS versus $\log R_{\mathrm{HK}}^{\prime}$ for the considered $T_{\text {eff }}$ (obtained using a polynomial fit on the curve shown in Fig. 5, second panel), $\mathrm{RV}_{\text {convbl } \odot}$ and $\mathrm{TSS}_{\odot}$ are the solar values. We also took into account the fact that stellar inclinations are expected to significantly affect the observed $\Delta R V_{\text {conv }}$. There is a general agreement in the trend with observations. The reconstructed values tend to be on average slightly higher than the observed values, although for most points this is within the $3 \sigma$ uncertainties. There are very few outliers above, which means that our star selection is good (we did not include stars with RV variations that cannot be explained by the $\log R_{\mathrm{HK}}^{\prime}$ behavior).

However, we still note a few stars for which we would have expected a larger RV amplitude given their convection level and activity variability. The most noticeable is HD 196390 (G1, $\Delta \log R_{\mathrm{HK}}^{\prime}=0.18$, lower right corner of the plot), which we classified into category 2 : it shows a very well defined and largeamplitude activity variation, and the RV variation, although correlated (correlation factor of 0.58), is indeed noisy. The slope of a linear fit of the RV versus $\log R_{\mathrm{HK}}^{\prime}$ multiplied by the observed $\Delta \log R_{\mathrm{HK}}^{\prime}$ leads to an RV amplitude of $15 \mathrm{~m} / \mathrm{s}$, which is closer to the expected value. This illustrates the limits of the analysis and the effect of the sampling on the estimations of $\Delta \log R_{\mathrm{HK}}^{\prime}$ and $\Delta \mathrm{RV}$, in addition to the intrinsic dispersion that is due to inclination. We therefore also tested another estimation of the observed $\Delta \mathrm{RV}$ by multiplying the slope RV versus $\log R_{\mathrm{HK}}^{\prime}$ for each star with $\Delta \log R_{\mathrm{HK}}^{\prime}$ : this gives similar results, although there are fewer outliers (such as HD 196390 or stars with a negative $\Delta \mathrm{RV}$ ). The comparison between observed and predicted $\triangle \mathrm{RV}$ therefore remains difficult.

The effect of inclination mentioned above refers to the difference in long-term amplitude of the variations of $\log R_{\mathrm{HK}}^{\prime}$ and $\mathrm{RV}_{\text {conv }}$ when a star like the Sun is seen from different angles, as shown by Borgniet et al. (2015): because the activity belt is located close to the equator, both amplitudes decrease when we move from a view from the equator to a pole-on point of view for a solar-type activity pattern. However, the longterm amplitude of $\mathrm{RV}_{\text {conv }}$ decreases faster because it cumulates two projection effects: the apparent size of the structure decreases toward the limb, and the projection of the convective blueshift along the line of sight decreases as well. In principle, another effect could be taken into account, that is, the effect of a variable convective blueshift with latitude (independently of magnetic activity), which is neglected here: solar granulation does not show strong variability with latitude, however (Rodriguez Hidalgo et al. 1992), and any variation seems to be mostly associated with magnetic structures (taken into account in the simulation). Therefore we do not expect the convective blueshift itself to vary significantly with inclination. We also note that we used a single law describing the relationship between activity level and TSS, which corresponds to the average inclination in our sample. Stars with similar average activity level but different inclinations should appear at a different position in the plots of Fig. 4 for example: compared to a pole-on observation, a star with a different inclination will appear at a lower activity level (if the activity belt is close to the equator, as for the Sun) and a higher |TSS| value as well, possibly with a proportionaly weaker attenuation given the projection effects. It is therefore possible that the TSS-Log $R_{\mathrm{HK}}^{\prime}$ laws exhibit slightly different slopes depending on the inclination, and they therefore contribute to the observed dispersion.

Finally, we note that our sample includes mostly G stars, and as shown in the figure, there are very few $\mathrm{K}$ stars. We find that they lie below the $\mathrm{G}$ stars for a given $\log R_{\mathrm{HK}}^{\prime}$ (orange circles in the lower panel of Fig. 9), which is expected. The F stars lie at the top, but not above, the G stars, showing that the flattened part at high $T_{\text {eff }}$ in the slope versus activity (Fig. 5, second panel) may be real.

\section{Conclusion}

We have analyzed a sample of 360 main-sequence stars with $T_{\text {eff }}$ between 4600 and $6400 \mathrm{~K}$ (K4 to F7), biased toward old stars, but also including a few relatively young very active stars (younger than $2 \mathrm{Gyr}$ ), and derived the amplitude of the convective blueshift for each star using the differential shift of spectral lines as in Paper I. We obtained the following results:

- We confirm the strong variation in convective blueshift with $T_{\text {eff }}$ obtained in Paper I. We observe a saturation toward lowmass stars.

- We confirm the dependence on the activity level. The attenuation factor of convection with activity seems to increase with $T_{\text {eff }}$ for $T_{\text {eff }}$ in the range $5200-6300 \mathrm{~K}$, although it is not possible to identify any trend for $T_{\text {eff }}$ below this range. In the smaller sample of Paper I, no trend was identified, which was in good agreement with the numerical simulation of Steiner et al. (2014) and Beeck et al. (2015), showing a similar magnetic field strength in magnetic structures for the different spectral types. If the magnetic field remains constant, it remains to be understood why the attenuation would be more efficient for more massive stars.

- We observe no significant difference between stars with a cycle and those without a cycle, suggesting similar properties of their active regions. 
- Similarly, we have not been able to identify a significant difference between the youngest stars in our sample (younger than 2 Gyr) and the other stars, also suggesting that plages are not very different.

- On the other hand, we observe a significant effect of metallicity, at least for solar-type stars for which we have a large sample: we estimate that stars with a high metallicity exhibit a lower convection level (as measured by the TSS and the convective blueshift) than those with a low metallicity, on the order of $10 \%$ for a $\Delta[\mathrm{Fe} / \mathrm{H}]$ of 0.27 , which is at least four times higher than predicted by numerical simulations. We also confirmed the dependence of the $\log R_{\mathrm{HK}}^{\prime}$ on metallicity observed by Jenkins et al. (2008) and discussed the possible effect on the possible relationship between metallicity and exoplanet that is due to biases in exoplanet surveys.

- We computed a percentage of $71 \%$ of stars that follow the "solar pattern", that is, stars that exhibit a good correlation between activity and RV variability, including stars with no variability for either variables, or stars with a low RV variability but a high $\log R_{\mathrm{HK}}^{\prime}$ variability (which could be due to inclination effects). Keeping in mind that the sample might be biased, we estimate that three quarters of the stars follow this pattern. A quarter of the stars exhibits high RV variations, however, which cannot be attributed to the inhibition of convection and which are probably due to other processes.

Acknowledgements. This work has been funded by the Université Grenoble Alpes project call "Alpes Grenoble Innovation Recherche (AGIR)" and on the ANR GIPSE ANR-14-CE33-0018. This work made use of several public archives and databases: The HARPS data have been retrieved from the ESO archive at http: //archive. eso.org/wdb/wdb/adp/phase3 spectral/form. This research has made use of the SIMBAD database, oper ated at the CDS, Strasbourg, France. Exoplanet information has been retrieved from the Extrasolar Planet Encyclopaedia at http://exoplanet.eu/. The solar spectrum from Kurucz et al. (1984) is available online at http://kurucz. harvard.edu/sun/fluxatlas2005/.

\section{References}

Allende Prieto, C., \& Lambert, D. L. 1999, A\&A, 352, 555

Allende Prieto, C., Lambert, D. L., Tull, R. G., \& MacQueen, P. J. 2002, ApJ, 566, L93

Allende Prieto, C., Koesterke, L., Ludwig, H.-G., Freytag, B., \& Caffau, E. 2013 A\&A, 550, A103

Beeck, B., Schüssler, M., Cameron, R. H., \& Reiners, A. 2015, A\&A, 581, A42

Borgniet, S. 2015, Ph.D. Thesis, Université Grenoble Alpes

Borgniet, S., Meunier, N., \& Lagrange, A.-M. 2015, A\&A, 581, A133

Borgniet, S., Lagrange, A.-M., Meunier, N., \& Galland, F. 2017, A\&A, 599, A57

Datson, J., Flynn, C., \& Portinari, L. 2014, MNRAS, 439, 1028

Delgado Mena, E., Bertrán de Lis, S., Adibekyan, V. Z., et al. 2015, A\&A, 576, A69 dos Santos, L. A., Meléndez, J., do Nascimento, J.-D., et al. 2016, A\&A, 592, A156

Dravins, D. 1987, A\&A, 172, 211

Dravins, D. 1999, in Precise Stellar Radial Velocities, eds. J. B. Hearnshaw, \&

C. D. Scarfe, ASP Conf. Ser., 185, IAU Colloq., 170, 268

Dravins, D. 2008, A\&A, 492, 199

Dravins, D., Lindegren, L., \& Nordlund, A. 1981, A\&A, 96, 345

Dravins, D., Larsson, B., \& Nordlund, A. 1986, A\&A, 158, 83

Fischer, D. A., \& Valenti, J. 2005, ApJ, 622, 1102

Fridlund, M., Hébrard, G., Alonso, R., et al. 2010, A\&A, 512, A14

Gray, D. F. 1982, ApJ, 255, 200

Gray, D. F. 1986, PASP, 98, 319

Gray, D. F. 2009, ApJ, 697, 1032

Gray, R. O., Corbally, C. J., Garrison, R. F., et al. 2006, AJ, 132, 161

Gray, R. O., Saken, J. M., Corbally, C. J., et al. 2015, AJ, 150, 203

Hamilton, D., \& Lester, J. B. 1999, PASP, 111, 1132

Holmberg, J., Nordström, B., \& Andersen, J. 2009, A\&A, 501, 941

Isaacson, H., \& Fischer, D. 2010, ApJ, 725, 875

Jenkins, J. S., Jones, H. R. A., Pavlenko, Y., et al. 2008, A\&A, 485, 571

Jenkins, J. S., Murgas, F., Rojo, P., et al. 2011, A\&A, 531, A8

Judge, P. G., \& Saar, S. H. 2007, ApJ, 663, 643

Kurucz, R. L., Furenlid, I., Brault, J., \& Testerman, L. 1984, Solar flux atlas from 296 to $1300 \mathrm{~nm}$ (New Mexico: National Solar Observatory)

Lagrange, A.-M., Meunier, N., Chauvin, G., et al. 2013, A\&A, 559, A83

Landstreet, J. D. 2007, in The Future of Photometric, Spectrophotometric and Polarimetric Standardization, ed. C. Sterken, ASP Conf. Ser., 364, 481

Lockwood, G. W., Skiff, B. A., Henry, G. W., et al. 2007, ApJS, 171, 260

Lovis, C., Mayor, M., Bouchy, F., et al. 2005, A\&A, 437, 1121

Lovis, C., Dumusque, X., Santos, N. C., et al. 2011, ArXiv e-prints [arXiv: 1107.5325$]$

Magic, Z., Collet, R., Asplund, M., et al. 2013, A\&A, 557, A26

Magic, Z., Collet, R., \& Asplund, M. 2014, ArXiv e-prints [arXiv: 1403.6245]

Mamajek, E. E., \& Hillenbrand, L. A. 2008, ApJ, 687, 1264

Marsden, S. C., Petit, P., Jeffers, S. V., et al. 2014, MNRAS, 444, 3517

Meunier, N., Desort, M., \& Lagrange, A.-M. 2010, A\&A, 512, A39

Meunier, N., Lagrange, A.-M., Mbemba Kabuiku, L., et al. 2017, A\&A, 597, A52

Mittag, M., Schmitt, J. H. M. M., \& Schröder, K.-P. 2013, A\&A, 549, A117

Nave, G., Johansson, S., Learner, R. C. M., Thorne, A. P., \& Brault, J. W. 1994, ApJS, 94, 221

Nordström, B., Mayor, M., Andersen, J., et al. 2004, A\&A, 418, 989

Perryman, M. A. C., Brown, A. G. A., Lebreton, Y., et al. 1998, A\&A, 331, 81

Ramírez, I., Meléndez, J., Bean, J., et al. 2014, A\&A, 572, A48

Reiners, A., Mrotzek, N., Lemke, U., Hinrichs, J., \& Reinsch, K. 2016, A\&A, 587, A65

Rocha-Pinto, H. J., \& Maciel, W. J. 1998, MNRAS, 298, 332

Rodriguez Hidalgo, I., Collados, M., \& Vazquez, M. 1992, A\&A, 264, 661

Santos, N. C., Israelian, G., Mayor, M., Rebolo, R., \& Udry, S. 2003, A\&A, 398, 363

Sousa, S. G., Santos, N. C., Mayor, M., et al. 2008, A\&A, 487, 373

Steiner, O., Salhab, R., Freytag, B., et al. 2014, PASJ, 66, S5

Strassmeier, K., Washuettl, A., Granzer, T., Scheck, M., \& Weber, M. 2000, A\&AS, 142, 275

Suárez Mascareño, A., Rebolo, R., González Hernández, J. I., \& Esposito, M. 2017, MNRAS, 468, 472

Tremblay, P.-E., Ludwig, H.-G., Freytag, B., Steffen, M., \& Caffau, E. 2013, A\&A, 557, A7

Valenti, J. A., \& Fischer, D. A. 2005, ApJS, 159, 141

Wright, J. T. 2004, AJ, 128, 1273 


\section{Appendix A: Additional table}

Table A.1. Star properties.

\begin{tabular}{|c|c|c|c|c|c|c|c|c|c|c|c|c|}
\hline Name & $T_{\text {eff }}$ & $B-V$ & $\begin{array}{l}\text { Sp. T. } \\
(\mathrm{K})\end{array}$ & $N_{\text {Spectra }}$ & $\begin{array}{l}\text { TSS } \\
(\mathrm{m} / \mathrm{s} /(\mathrm{F} / \mathrm{Fc}))\end{array}$ & $\begin{array}{l}\sigma \mathrm{TSS} \\
(\mathrm{m} / \mathrm{s} /(\mathrm{F} / \mathrm{Fc}))\end{array}$ & $\log R_{\mathrm{HK}}^{\prime}$ & $\begin{array}{l}\sigma \\
\log R_{\mathrm{HK}}^{\prime}\end{array}$ & $\begin{array}{l}\text { Conv. } \\
\text { Blueshift } \\
(\mathrm{m} / \mathrm{s})\end{array}$ & $\begin{array}{l}\text { Age } \\
\text { Gyr }\end{array}$ & $\begin{array}{l}v \sin i \\
\mathrm{~km} \mathrm{~s}^{-1}\end{array}$ & Source \\
\hline HD 169830 & $6361^{1}$ & F7 & 0.47 & 65 & -1102.6 & 21.5 & -4.976 & 0.003 & -504.4 & $2.30^{10}$ & $4^{13}$ & 1 \\
\hline HD 184985 & $6362^{8}$ & F7 & 0.45 & 38 & -1148.4 & 22.1 & -4.957 & 0.002 & -525.4 & $2.40^{10}$ & $5^{13}$ & 5 \\
\hline HD 693 & $6297^{8}$ & F8 & 0.49 & 35 & -1078.7 & 35.3 & -4.940 & 0.002 & -493.5 & $3.80^{10}$ & $5^{13}$ & 5 \\
\hline HD 11226 & $6098^{1}$ & F8 & 0.58 & 38 & -999.6 & 15.4 & -5.002 & 0.004 & -457.3 & $4.20^{10}$ & $3^{13}$ & 1 \\
\hline HD 38382 & $6082^{1}$ & F8 & 0.53 & 29 & -904.6 & 14.9 & -4.908 & 0.004 & -413.8 & $2.10^{10}$ & $3^{13}$ & 1 \\
\hline
\end{tabular}

Notes. This table is published in its entirety at the CDS. A portion is shown here for guidance regarding its form and content. Star name, spectral type and $B-V$ (both from the CDS), $T_{\text {eff }}$, number of spectra used in the analysis, TSS and its $1 \sigma$ uncertainty, averaged Log $R_{\mathrm{HK}}^{\prime}$ and its $1 \sigma$ uncertainty, convective blueshift derived from the TSS, age, $v \sin i$, and origin (survey) of the observations. The origins of the observations (references 1 to 7) are defined in Table 1.

References. 1, Sousa et al. (2008); 2, Ramírez et al. (2014); 3, Marsden et al. (2014); 4, Datson et al. (2014); 5, Borgniet et al. (2017); 6, Lagrange et al. (2013); and 7, Gray et al. (2015). The sources for $T_{\text {eff }}$, age, and $v \sin i$ are either from these references (1-7) or from 8, Gray et al. (2006); 9, Allende Prieto \& Lambert (1999); 10, Holmberg et al. (2009); 11, Delgado Mena et al. (2015); 12, Borgniet (2015); 13, Nordström et al. (2004); 14, Jenkins et al. (2011); 15, Valenti \& Fischer (2005); 16, dos Santos et al. (2016); 17, Strassmeier et al. (2000); and 18, Lovis et al. (2005).

\section{Appendix B: Metallicity effect}

To quantify the difference in TSS for stars of different metallicity as shown in Sect. 3.4 (see Fig. 8), we computed the linear law fitting the TSS versus $\log R_{\mathrm{HK}}^{\prime}$ only for stars with a negative metallicity (average $[\mathrm{Fe} / \mathrm{H}]$ of -0.19 , or -0.13 when restricted to $\log R_{\mathrm{HK}}^{\prime}$ lower than -4.95$)$. Then, below -4.95 , we estimated the average TSS that stars with a positive metallicity would have if they were following this linear law, compared to the observed value: this gives $-754 \mathrm{~m} / \mathrm{s}$ and $-877 \mathrm{~m} / \mathrm{s}$, respectively. The same procedure was applied to the convective blueshift and gives -401 and $-345 \mathrm{~m} / \mathrm{s}$ respectively, hence a difference of $56 \mathrm{~m} / \mathrm{s}(14 \%$ with respect to the linear law) for a $\Delta[\mathrm{Fe} / \mathrm{H}]$ of 0.27 . The effect is similar in the $T_{\text {eff }}$ range $5300-5662 \mathrm{~K}$, with a difference of 38 $\mathrm{m} / \mathrm{s}$ on the convective blueshift representing $12 \%$ for a $\Delta[\mathrm{Fe} / \mathrm{H}]$ of 0.32 .
When we assume that for $\log R_{\mathrm{HK}}^{\prime}$ below -4.80 , the observed trend of $\log R_{\mathrm{HK}}^{\prime}$ versus $[\mathrm{Fe} / \mathrm{H}]$ is entirely due to the effect of the metallicity on the $\log R_{\mathrm{HK}}^{\prime}$ measurement (and not an intrinsic variation), we can correct the $\log R_{\mathrm{HK}}^{\prime}$ values for this trend and again apply the same procedure. For the $T_{\text {eff }}$ range $5662-$ $5918 \mathrm{~K}$, this gives a convective blueshift of $-345 \mathrm{~m} / \mathrm{s}$ (average) and -385 (from the negative metallicity linear fit), hence a difference of $40 \mathrm{~m} / \mathrm{s}$ representing $10 \%$ : this is slightly smaller than before correction, but on the same order of magnitude, so this result seems to be robust. We also note that Rocha-Pinto \& Maciel (1998) studied the difference between the chromospheric and isochrone ages, which would in principle also provide a way to correct for the effect of metallicity on $\log R_{\mathrm{HK}}^{\prime}$ measurements, but the resulting correction seems unrealistic, which could be due to the very large uncertainties on age estimations. 